\title{
miR-486-5p suppresses gastric cancer cell growth and migration through downregulation of fibroblast growth factor 9
}

\author{
WEIWEI WEI ${ }^{1 *}$, CHUNYU LIU $^{1 *}$, RONGRONG YAO $^{2}$, QINGYUN TAN $^{3}$, \\ QINGDONG WANG ${ }^{3}$ and HAO TIAN ${ }^{1}$ \\ Departments of ${ }^{1}$ General Surgery, ${ }^{2}$ Interventional Radiology and ${ }^{3}$ Anesthesiology, \\ First Affiliated Hospital of Jiamusi University, Jiamusi, Heilongjiang 154002, P.R. China
}

Received April 2, 2021; Accepted August 26, 2021

DOI: $10.3892 / \mathrm{mmr} .2021 .12411$

\begin{abstract}
Non-coding RNAs serve essential roles in regulating mRNA and protein expression and dysregulation of non-coding RNAs participates in a variety of types of cancer. microRNAs (miRNAs/miRs), which are 21-24 nucleotides non-coding RNAs, have been shown to be important for the development of gastric cancer (GC). However, the role of miR-486-5p in $\mathrm{GC}$ remains to be elucidated. The present study found that miR-486-5p was downregulated in GC tissues. Comparing with gastric normal cells GES-1, GC cells, including MKN-45, AGS, HGC27 and MKN74, had reduced abundance of miR-486-5p transcript. CCK8 and colony formation assays demonstrated that GC cell growth and proliferation were enhanced by miR-486-5p inhibitors and were suppressed by miR-486-5p mimics. miR-486-5p also suppressed cell cycle process and migration and promoted apoptosis in GC cells, as verified by propidium iodide (PI) staining, Transwell assay and PI/Annexin V staining. miR-486-5p downregulated fibroblast growth factor 9 (FGF9) through combining to its 3'untranslated region. Overexpression of FGF9 accelerated the growth and proliferation of GC cells. The expression of miR-486-5p was negatively associated with FGF9 mRNA expression in GC samples. These results revealed that miR-486-5p was a tumor suppressor in GC. Downregulation of FGF9 contributed to the role of miR-486-5p in GC.
\end{abstract}

Correspondence to: Dr Qingdong Wang, Department of Anesthesiology, First Affiliated Hospital of Jiamusi University, 348 Dexiang Street, Jiamusi, Heilongjiang 154002, P.R. China

E-mail: jmsu_east@163.com

Dr Hao Tian, Department of General Surgery, First Affiliated Hospital of Jiamusi University, 348 Dexiang Street, Jiamusi, Heilongjiang 154002, P.R. China

E-mail: tianhaolove@126.com

${ }^{*}$ Contributed equally

Key words: gastric cancer, miR-486-5p, fibroblast growth factor 9, cell growth, cell migration

\section{Introduction}

GC is the fifth commonest malignancy and the third leading cause of cancer-related mortality in the world (1). According to the World Health Organization, GC is classified into several subtypes regarding to the molecular pathogenesis and clinicopathological profiles, including papillary, tubular and mucinous adenocarcinomas, mixed carcinomas and poorly cohesive carcinomas, with or without a component of ring cells (2).H.pylori infection, which causes chronic inflammation in gastric and subsequent cancer development, is the major risk factor for GC (3). Due to vaccination or drugs developed to treat $H$. pylori infection, the incidence of $H$. pylori-related GC has decreased, whereas genetic variation-induced GC patients has increased (4). Accumulating evidence has identified that TP53, PIK3CA and APC are the most frequent mutated genes in GC patients (5-7). Additionally, there are potentially amplifications of receptor tyrosine kinases, including Erb-B2 Receptor Tyrosine Kinase 2 (ERBB2) and EGFR, also common in solid tumors (8). However, there is a lack of effective drugs to treat the patients carrying these alterations. Novel disease avenues need to be explored for clinical diagnosis and treatment.

microRNAs (miRNAs), which contain 21-24 nucleotides, are types of non-coding RNA. They control different cellular functions by targeting the abundance of the mRNA of key genes in cells (9). Dysregulation of miRNAs stimulates or suppresses cancer growth, proliferation and metastasis by downregulating tumor suppressors or oncogenes (10). Well-known oncogenic miRNAs are miR-17 and miR-21. For example, cancer-associated fibroblasts secrete miR-17, which enhances the aggressiveness of colon cancer by regulating the Runt-related transcription factor (RUNX)3/Myc/TGF- $\beta 1$ cascade (11). miR-17-5p is overexpressed in GC patients (12). Upregulation of miR-17-5p contributes to GC growth and metastasis by downregulating RUNX3 (13). In addition, the oncogenic function of miR-17 has been reported in thyroid cancer, laryngeal squamous cell carcinoma, breast cancer and prostate cancer (14-16). miR-21 is highly expressed in a wide range of types of cancer, such as lung cancer, GC, colon cancer and prostate cancer (17-20). miR-21 in serum is considered as a marker of colorectal cancer (CRC) and its high expression is associated with shorter progression free survival of CRC patients. As well as miR-21, various miRNAs, including 
miR-20a-5p, miR-103a-3p, miR-106a-5p and miR-143-5p, have been demonstrated to act as novel biomarkers for CRC recurrence (21). miR-21 promotes the growth and metastasis of various types of cancer by targeting different tumor suppressors, including SMAD7, PTEN and HMG-box transcription factor 1 (22). As a previous study indicated, PTEN deficiency, which might be associated with gene mutations, loss of heterozygosity and promoter hypermethylation, serves as an indicator of the pathological state of GC. When considered with ERBB2 expression, PTEN deficiency was related to a more aggressive proliferative capacity of tumors (23).

miR-486-5p was initially identified as a tumor suppressive microRNA in lung cancer; Pang et al (24) found that among 26 miRNAs, miR-486-5p is one of the most significantly decreased miRNAs in non-small-cell lung cancer. A subsequent study showed that miR-486-5p is downregulated in breast cancer patients (25). miR-486-5p also suppresses the growth and proliferation of papillary thyroid carcinoma cells by negatively regulating fibrillin-1 (26).

The present study investigated the expression of miR-486-5p in GC tissues by analyzing The Cancer Genome Atlas (TCGA) database (http://tcga-data.nci.nih.gov/tcga/) and reverse transcription-quantitative (RT-q) PCR in samples of patients. Overexpression by mimics and knockdown by inhibitors were used to study the role of miR-486-5p in GC cell function based on CCK8, colony formation, cell cycle analysis, apoptosis measurement and Transwell analysis of migration. The findings suggested that miR-486-5p is a tumor suppressor in GC.

\section{Materials and methods}

Cell lines and regents. Gastric normal cells GES-1 and GC cells MKN-45, AGS, HGC27 and MKN74 were obtained from the American Type Culture Collection. miR-486-5p mimics, inhibitors and their controls were purchased from Guangzhou RiboBio Co., Ltd. The CCK8 kit and cell cycle assay kit were from Beyotime Institute of Biotechnology. The apoptosis assay kit was from Invitrogen (Thermo Fisher Scientific, Inc.).

Clinicalanalysis of miR-486-5p. The expression of miR-486-5p was analyzed in GC and normal tissues based on The Cancer Genome Atlas (http://cancergenome.nih.gov) website and the samples collected from First Affiliated Hospital of Jiamusi University hospital between April 2019 and October 2019. A total of 372 cancer and 32 normal tissues from TCGA database were used to analyze miR-486-5p. A total of 23 cancer and 23 normal tissues (5 $\mathrm{cm}$ distal to the cancer tissues) were collected from GC patients by surgery (male:female, 10:13; age range: 30-65 years old). The tissues were harvested before any drug interventions. The expression of miR-486-5p between 23 cancer and 23 normal tissues was compared using paired Student's t-test. A written informed consent was obtained from each patient. The experiments were carried out according to World Medical Association Declaration of Helsinki and were supported by the Ethics Committee of First Affiliated Hospital of Jiamusi University (Ethic approval no. 202067).

Cell culture. GES-1, MKN-45, AGS, HGC27 and MKN74 cells were grown in Dulbecco modified Eagle's medium (DMEM) or RPMI-1640 (Corning, Inc.), which was supplemented with
10\% fetal bovine serum (Gibco; Thermo Fisher Scientific, Inc.) and $1 \%$ penicillin/streptomycin antibiotics (Gibco; Thermo Fisher Scientific, Inc.). Cells were cultured at $37^{\circ} \mathrm{C}$ containing $5 \% \mathrm{CO}_{2}$.

miR-486-5p downregulation and overexpression. To knock down and to overexpress miR-486-5p, miR-486-5p inhibitors $(40 \mathrm{nM})$, mimics $(40 \mathrm{nM})$ and their controls $(40 \mathrm{nM})$ were used to transfect AGS and HGC27 cells ( $80 \%$ confluence) by using Lipofectamine 2000 (Invitrogen; Thermo Fisher Scientific, Inc.). After $48 \mathrm{~h}$ at $37^{\circ} \mathrm{C}$, the cells were used for CCK8, colony formation, cell cycle, apoptosis, migration, RT-qPCR and immunoblotting analysis. The sequence of miR-486-5p mimics and inhibitor were: miR-486-5p mimics: sense: UCCUGU ACUGAGCUGCCCCGAG, antisense: CUCGGGGCAGCU CAGUACAGGA; miR-486-5p inhibitor: CUCGGGGCAGCU CAGUACAGGA; miR-486-5p mimics control: sense: UUU GUACUACACAAAAGUACUG, antisense: CAGUACUUU UGUGUAGUACAAA; miR-486-5p inhibitor control: CAG UACUUUUGUGUAGUACAAA. The mimics, inhibitor and controls were purchased from Huzhou Hippo Biotechnology Co., Ltd.

Ectopic expression of FGF9 by lentivirus. The coding sequence of FGF9 (cat. no. NM_002010) was synthesized by Tianyi Huiyuan Biotech Co., Ltd. and then cloned into overexpressing GV115 lentivirus vectors (Shanghai GeneChem). The GV115 $(20 \mu \mathrm{g})$, pHelper1.0 $(15 \mu \mathrm{g})$ and pHelper2.0 $(10 \mu \mathrm{g})$ were cotransfected into $293 \mathrm{~T}$ cells at $37^{\circ} \mathrm{C}$ for $6 \mathrm{~h}$ using Lipofectamine ${ }^{\circledR} 3000$ (Invitrogen; Thermo Fisher Scientific, Inc.). The virus were concentrated and used to infect AGS and $\mathrm{HGC} 27$ cells when cell density reached $70 \%$ confluence. The subsequent transfection with the lentiviruses was for $72 \mathrm{~h}$ $(\mathrm{MOI}=10)$ at $37^{\circ} \mathrm{C}$.

RNA isolation and RT-qPCR. Human GC, normal tissues and cells were collected and homogenized in TRIzol ${ }^{\circledR}$ (Thermo Fisher Scientific, Inc.). Total RNA of $100 \mathrm{mg}$ tissues or $1 \times 10^{6}$ cells was extracted from the tissues following the manufacturer's protocols. RNA to cDNA conversion was performed using miRNA First Strand cDNA Synthesis kit (Vazyme Biotech Co., Ltd.), according to the manufacturer's protocols. Quantification of miRNA was determined by using SYBR Master Mixture (TransGen Biotech Co., Ltd.) on the Bio-Rad qPCR system (Bio-Rad Laboratories, Inc.), according to the manufacturer's protocols. The following thermocycling conditions were used for qPCR: Initial denaturation at $95^{\circ} \mathrm{C}$ for $30 \mathrm{sec}$; followed by 40 cycles at $95^{\circ} \mathrm{C}$ for $10 \mathrm{sec}, 60^{\circ} \mathrm{C}$ for $30 \mathrm{sec}$; lastly $95^{\circ} \mathrm{C}$ for $25 \mathrm{sec}, 60^{\circ} \mathrm{C}$ for $60 \mathrm{sec}, 95^{\circ} \mathrm{C}$ for $15 \mathrm{sec}$. Relative expression levels were calculated using the $2^{-\Delta \Delta \mathrm{Cq}}$ method (27). The experiments were performed three times. U6 was used as an internal control. Primer sequences were: $\mathrm{miR}-486-5 \mathrm{p}$ forward: 5'-GCCGTCCTGTCATGAGCTGC-3' and reverse: 5'-GTGCAGGGTCCGAGGT-3'; U6 forward: 5'-CTCGCT TCGGCAGCACATATACT-3' and reverse: 5'-ACGCTTCAC GAATTTGCGTGTC-3'.

Western blotting. Total proteins were extracted from the cells using the RIPA lysis buffer (Beyotime Institute of Biotechnology). The concentration of total protein was 
detected using a BCA assay kit (Thermo Fisher Scientific, Inc.) Equal amount of the proteins $(30 \mu \mathrm{g})$ were separated on 10-12\% SDS-PAGE gels and transferred onto PVDF membranes. The membranes were blocked by $5 \%$ non-fat milk dissolved in PBST for $1 \mathrm{~h}$ at room temperature and incubated with indicated primary antibodies at $4^{\circ} \mathrm{C}$ overnight, including antibodies against E-cadherin (1:1,000; cat. no. 14472), $\mathrm{N}$-cadherin (1:1,000; cat. no. 13116), Vimentin $(1: 1,000$; cat. no. 5741) and cleaved caspase-3 (1:1,000; cat. no. 9661) were from Cell Signaling Technology, Inc. Antibody against FGF9 (1:1,000; cat. no. ab206408) was from Abcam. GAPDH (1:1,000; cat. no. 60004-1-Ig), $\beta$-actin $(1: 1,000$; cat. no. 66009-1-Ig). And the secondary antibodies, including (HRP-conjugated Affinipure Goat Anti-Mouse $\operatorname{IgG}(\mathrm{H}+\mathrm{L})$, 1:10,000; cat. no. SA00001-1; HRP-conjugated Affinipure Goat Anti-Mouse IgG (H+L), 1:10,000; cat. no. SA00001-1; HRP-conjugated Affinipure Goat Anti-Rabbit IgG (H+L), 1:10,000; cat. no. SA00001-2), obtained from ProteinTech Group, Inc., were incubated for $2 \mathrm{~h}$ at $4^{\circ} \mathrm{C}$. The protein was visualized using Pierce ECL Western Blotting substrate (Thermo Fisher Scientific, Inc.), and analyzed using Image Lab 6.1 software (Bio-Rad Laboratories, Inc.).

CCK8 assay. After transfecting GC cells with miR-486-5p, miR-486-5p inhibitors, mimics and their controls for $48 \mathrm{~h}$, the cells were counted and a total of 2,000 cells were seeded in triplicate into 96 -well plates and cultured at $37^{\circ} \mathrm{C}$ and $5 \% \mathrm{CO}_{2}$. At indicated time points, $10 \%$ of CCK 8 regent was incubated with culture medium for $3 \mathrm{~h}$ at $37^{\circ} \mathrm{C}$. Then, the plates were vibrated for $30 \mathrm{sec}$ and absorbance at $450 \mathrm{~nm}$ was detected on a microplate reader.

Colony formation assay. After transfecting GC cells with miR-486-5p, miR-486-5p inhibitors, mimics and their controls for $48 \mathrm{~h}$, the cells were counted and a total of 2,000 cells were seeded into 6 -well plates in triplicate and cultured at $37^{\circ} \mathrm{C}$ and $5 \% \mathrm{CO}_{2}$. Colonies were formed 10 days later and culture medium was removed. Then, the colonies were washed by PBS for three times and fixed by methanol for $30 \mathrm{~min}$ at room temperature. Following staining with $0.2 \%$ crystal violet (Beijing Solarbio Science \& Technology Co., Ltd.) for $30 \mathrm{~min}$ at room temperature, the cell colonies (>50 cells) were washed and images captured using a camera.

Cell cycle. Cell cycle was analyzed by using propidium iodide (PI) staining. After transfecting GC cells with miR-486-5p, miR-486-5p inhibitors, mimics and their controls for $48 \mathrm{~h}$, the cells were harvested by trypsin and were washed with iced PBS for three times. The cells were fixed in $70 \%$ ethanol overnight at $-20^{\circ} \mathrm{C}$. Then the cells were stained with PI at $37^{\circ} \mathrm{C}$ for $30 \mathrm{~min}$ and cell cycle was analyzed on a flow cytometry system (cytoFLEX, Beckman Coulter, Inc.). The data were analyzed by CytExpert (Version 2.4.0.28; Beckman Coulter, Inc.).

Apoptosis. Cell apoptosis was analyzed by using PI/Annexin V staining. After transfecting GC cells with miR-486-5p, miR-486-5p inhibitors, mimics and their controls for $48 \mathrm{~h}$, the cells were harvested by using EDTA-free trypsin. Then, the cells were washed by PBS and stained with PI and Annexin V for $15 \mathrm{~min}$ at room temperature. Immediately, cell apoptosis was analyzed on the flow cytometry system (cytoFLEX, Beckman Coulter, Inc.). CytExpert software (Version 2.4.0.28, Beckman Coulter, Inc.) was used for data analysis. The apoptosis rate was calculated as the percentage of early and late apoptotic cells.

Luciferase reporter assay. The wild-type or mutant 3'UTR of FGF9 was inserted into psi-CHECK vectors (Promega Corporation). The vectors were transfected into 293T cells using Lipofectamine ${ }^{\circledR} 2000$ (Invitrogen; Thermo Fisher Scientific, Inc.). $48 \mathrm{~h}$ later, luciferase activity was detected by using Dual-Luciferase Reporter Assay System (Promega Corporation). Renilla luciferase activity indicated transfection efficiency.

Statistical analysis. Statistical analysis was performed using SPSS 26 software (IBM Corp.). Students' t-test was performed to compare difference between two groups and one-way analysis of variance (ANOVA) followed by Tukey's post hoc test was applied for comparison between $\geq 3$ groups. Linear regression was performed to analysis correlation between FGF9 and miR-486-5p using the public database http://gepia. cancer-pku.cn/. $\mathrm{P}<0.05$ was considered to indicate a statistically significant difference.

\section{Results}

The expression of miR-486-5p is detected in GC tissues and cells. The expression of miR-486-5p in GC and normal tissues was examined by analyzing the data from TCGA database. The abundance of miR-486-5p was analyzed in a total of 372 stomach adenocarcinoma (STAD) and 32 normal tissues. It was found that miR-486-5p was downregulated in STAD tissues compared with normal tissues (Fig. 1A). Then, GC tissues and adjacent tissues were collected from the patients before any interventions. RT-qPCR analysis of miR-486-5p results found that miR-486-5p level was reduced in GC tissues comparing to normal tissues (Fig. 1B), which was consistent with the data analyzed from TCGA database. miR-486-5p level was decreased in GC cells, such as MKN-45, AGS, HGC27 and MKN74, comparing with gastric normal cells GES-1 (Fig. 1C). These results indicate that miR-486-5p is downregulated in GC tissues and cells.

miR-486-5p retards the proliferation ability of $G C$ cells. To study the function of miR-486-5p in GC, mimics and inhibitors were applied to overexpress and to downregulate miR-486-5p in GC cells. Due to the difficulties in performing the Transwell assay on MKN45 and MKN74 cells, AGS and HGC27 cells were used to in the present study. As RT-qPCR results suggested, the miR-486-5p mimics effectively overexpressed miR-486-5p expression in both AGS and HGC27 cells (Fig. 2A), while there were no obvious changes in miR-486-5p expression levels following transfection with miR-486-5p inhibitor (Fig. S1). CCK8 assay was performed to detect cell growth. The results showed that miR-486-5p overexpression by mimics suppressed the cell growth of AGS cells. Similar results were found in $\mathrm{HGC} 27$ cells after overexpressing miR-486-5p. By contrast, miR-486-5p downregulation enhanced the growth and proliferation capacity of AGS and 
A

hsa-miR-486-5p with 372 cancer and 32 normal samples in STAD

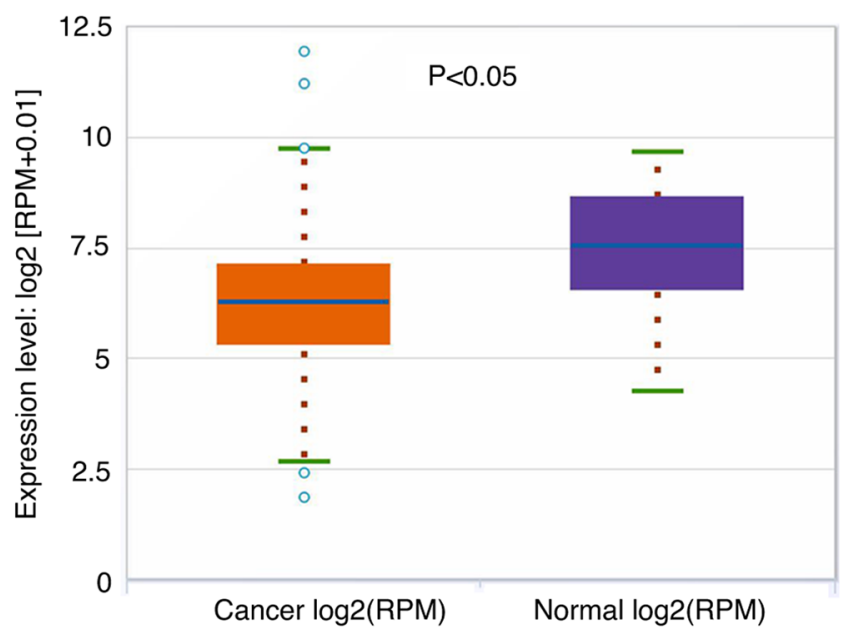

C 1.5
B

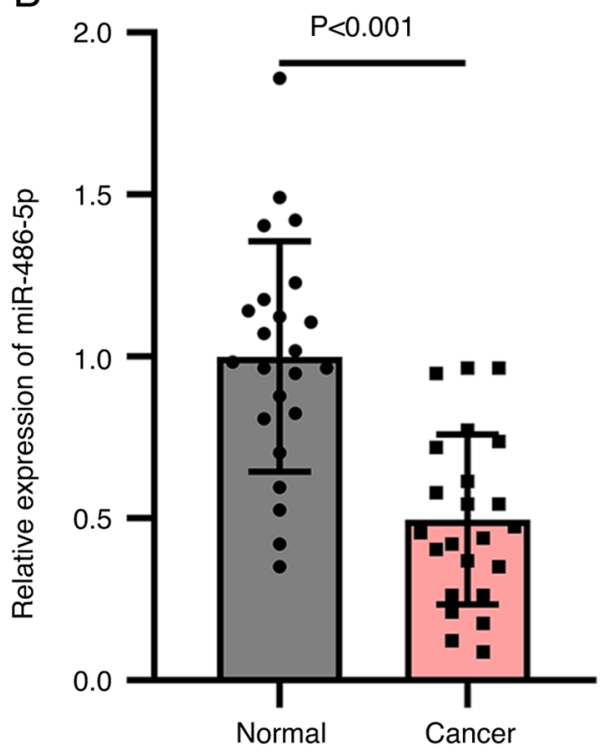

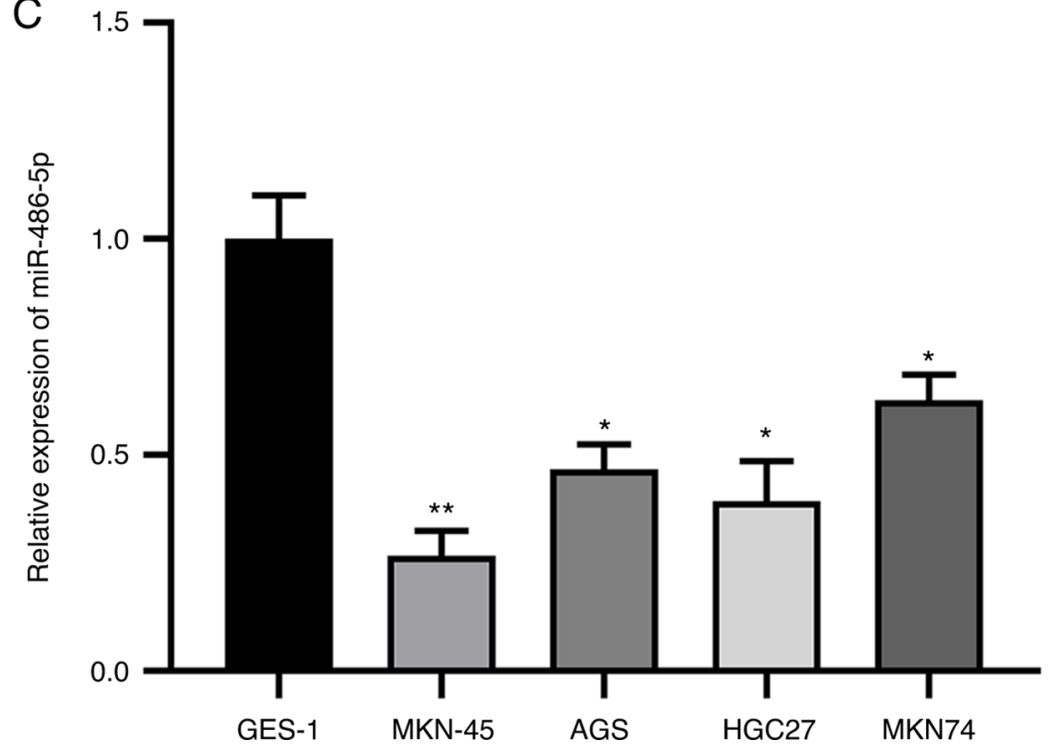

Figure 1. miR-486-5p expression is reduced in GC patients. (A) The expression of miR-486-5p was analyzed in stomach adenocarcinoma and normal tissues from The Cancer Genome Atlas database. (B) The expression of miR-486-5p was analyzed in GC and normal tissues based on reverse transcription-quantitative PCR. (C) The expression of miR-486-5p was analyzed in gastric normal cells GES-1 and GC cells, including MKN-45, AGS, HGC27 and MKC74. * $<0.05$, ${ }^{* *} \mathrm{P}<0.01$. miR, microRNA; GC, gastric cancer; RPM, reads per million.

HGC27 cells (Fig. 2B and C). miR-486-5p ectopic expression suppressed the colony formation ability of AGS and HGC27 cells. By contrast, miR-486-5p knockdown resulted in accelerated colony growth in AGS and HGC27 cells (Fig. 2D and E). Thus, miR-486-5p was a tumor suppressor in GC.

miR-486-5p induces cell apoptosis and cell cycle arrest at $G_{0} / G_{1}$ phase. Suppressed cell apoptosis is a hallmark of cancer (28). The present study next explored the effect of miR-486-5p on cell apoptosis in GC cells by staining the cells with PI/Annexin V-APC and flow cytometry analysis. The results showed that miR-486-5p mimics enhanced cell apoptosis in AGS and HGC27 cells, while opposite results were found in the cells which were transfected with miR-486-5p inhibitors (Fig. 3A and B). In addition, the expression of cleaved-caspase 3 was effectively upregulated following treatment with miR-486-5p mimics, while downregulated in AGS and HGC27 cells transfected with miR-486-5p inhibitors (Fig. 3C), which was consistent with the apoptosis detection results. It has been shown that miR-486-5p regulates cell cycle progress in lung cancer cells (29). To study the role of miR-486-5p in cell cycle in GC cells, the cells transfected with Ctrl-mimics, miR-486-5p mimics and miR-486-5p inhibitors were subjected to PI staining and flow cytometry analysis. It was found that miR-486-5p overexpression resulted in increased $G_{0} / G_{1}$ phase and decreased $G_{2} / M$ phase in cell cycle. By contrast, miR-486-5p downregulation showed adverse effect on cell cycle progress (Fig. 3D and E). Therefore, miR-486-5p regulated apoptosis and cell cycle in GC cells.

miR-486-5p suppresses migration and epithelial-to-mesenchymal transition in GC cells. Metastasis is a major problem in GC (30). Transwell assay demonstrated that miR-486-5p 
A

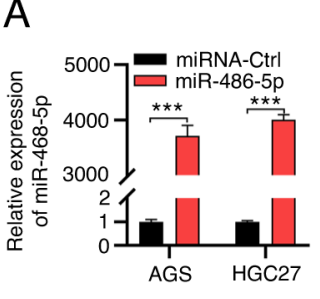

D

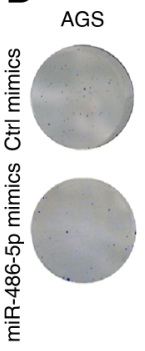

B

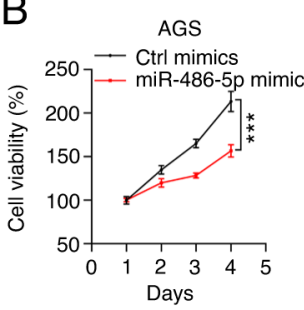

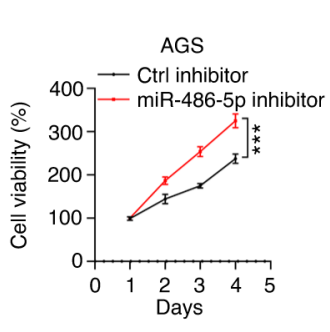

$E$
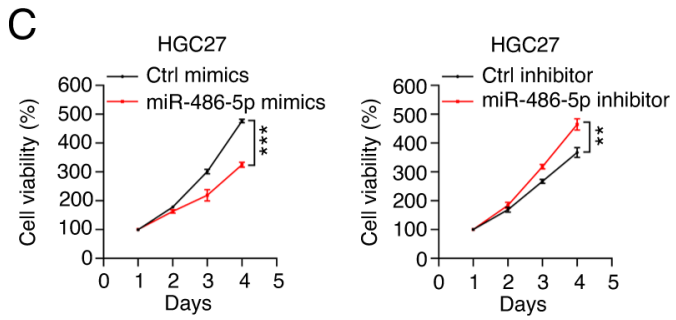
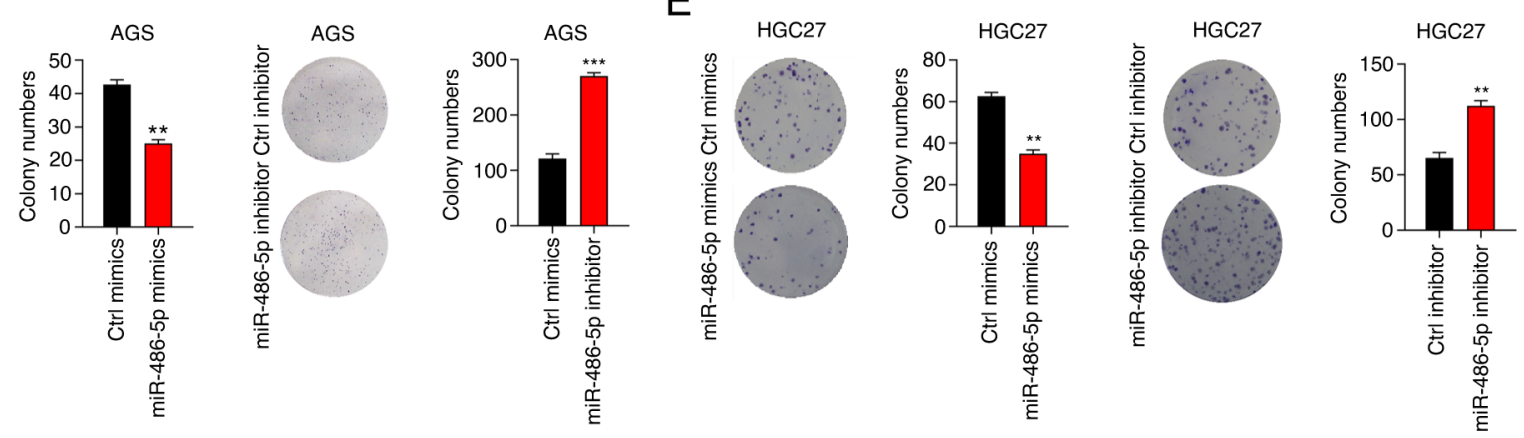

Figure 2. miR-486-5p suppresses GC growth and colony formation. (A) miR-486-5p expression was determined in AGS and HGC27 cells transfected with Ctrl mimics and miR-486-5p mimics using reverse transcription-quantitative PCR. CCK8 was performed to detect cell growth in (B) AGS and (C) HGC27 cells transfected Ctrl mimics, miR-486-5p mimics, Ctrl inhibitor and miR-486-5p inhibitors. ${ }^{* *} \mathrm{P}<0.01,{ }^{* * *} \mathrm{P}<0.001$. Colony formation was detected in (D) AGS and (E) HGC27 cells transfected with Ctrl mimics, miR-486-5p mimics, Ctrl inhibitor and miR-486-5p inhibitors. miR, microRNA; GC, gastric cancer; Ctrl, control.

upregulation by mimics reduced cell migration and invasion in AGS and HGC27 cells. By contrast, miR-486-5p downregulation increased the migration and invasion ability of AGS and HGC27 cells (Fig. 4A-D). Epithelial-to-mesenchymal transition (EMT) is a characteristic of metastasis (31). As immunoblotting results showed, miR-486-5p overexpression upregulated E-cadherin and downregulated $\mathrm{N}$-cadherin and Vimentin in AGS cells, while knockdown of miR-486-5p effectively inhibited E-cadherin, upregulated N-cadherin and Vimentin expression in HGC27 cells (Fig. 4E). Taken together, miR-486-5p inhibited migration, invasion and EMT in GC.

miR-486-5p downregulates FGF9 by binding to its 3'UTR sequence. To explore the downstream targets of miR-486-5p, the negatively correlated genes of miR-486-5p from TCGA database were analyzed and it was found that expression of FGF9 was inversely associated with expression of miR-486-5p (Fig. 5A). To validate whether miR-486-5p regulated FGF9, immunoblotting assays were performed to analyze FGF9 in Ctrl mimics and miR-486-5p overexpressed cells. The results showed that miR-486-5p overexpression suppressed the expression of FGF9 in GC cells (Fig. 5B). The target for miR-486-5p was analyzed by TargetScan and it was observed that FGF9 was a potential target for miR-486-5p (Fig. 5C). Luciferase activity was then assessed in 293T cells transfected with psi-CHECK containing NC, wild type or mutant 3'UTR sequence of FGF9. The results showed that miR-486-5p significantly decreased the luciferase activity in cells transfected with wide type 3'UTR but had no effect in cells transfected with NC or mutant vectors (Fig. 5D). Taken together the data suggested that miR-486-5p bound to the 3'UTR sequence to suppress FGF9 expression.
FGF9 restores cell function in miR-486-5p Overexpressed $G C$ cells. FGF9 has been shown to be an oncoprotein in cancers (32) and the role of FGF9 in GC should be determined. The present study overexpressed FGF9 by lentivirus in AGS and HGC27 cells transfected with miR-486-5p mimics. The results suggested that FGF9 was significantly overexpressed in AGS and HGC27 cells transfected with FGF9-overexpression vector (Fig. S2). Western blotting results demonstrated that FGF9 was efficiently downregulated following treatment with miR-486-5p mimics, while the expression of FGF9 was recovered in the cells transfected with miR-486-5p mimics (Fig. 6A). When miR-486-5p mimics suppressed the proliferation of AGS and HGC27 cells, FGF9 overexpression restored the cell proliferation capacity (Fig. 6B). Consistent results were observed in cell colony formation in AGS and HGC27 cells (Fig. 6C and D). Thus, it was concluded that miR-486-5p downregulation of FGF9 inhibited GC cell growth and proliferation.

\section{Discussion}

$\mathrm{GC}$ is one of the commonest malignancies and a large number of cancer patients succumb to this deadly disease. The present study found that miR-486-5p was downregulated in GC tissues. Loss-of-function and gain-of-function assays demonstrated that miR-486-5p acted as a tumor suppressive microRNA in GC, as revealed by CCK8, colony formation, apoptosis, cell cycle and migration assays. miR-486-5p also suppressed EMT in GC cells. Thus, miR-486-5p downregulation in GC patients may contribute to GC progression.

miR-486-5p was initially identified as a tumor suppressor in lung cancer. Pim-1 kinase oncogene was the target for miR-486-5p in lung cancer (24). In addition, downregulation 

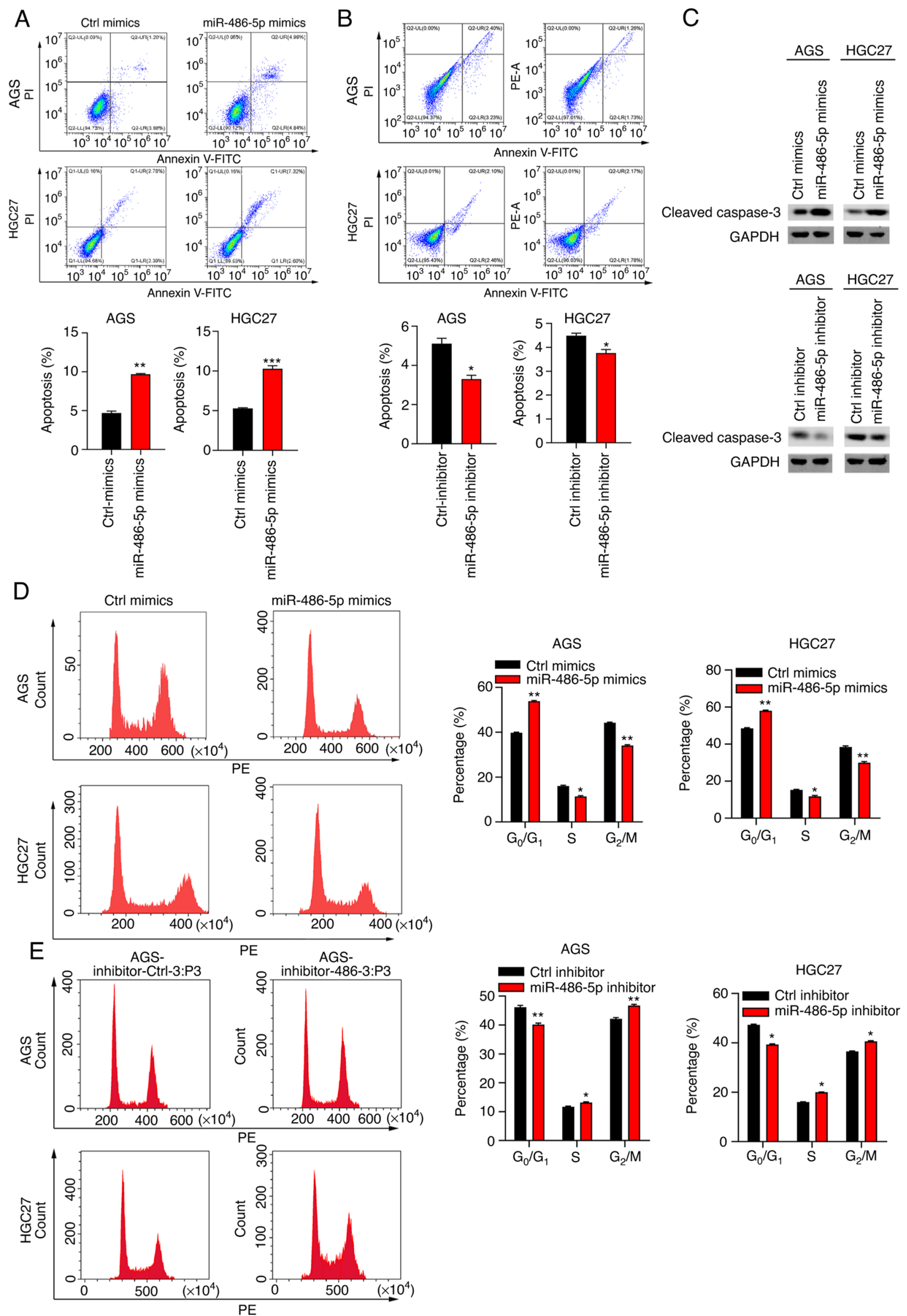

Figure 3. miR-486-5p promotes apoptosis and cell cycle arrest in GC cells. (A and B) Cell apoptosis was detected by PI/Annexin V staining in AGS and HGC27 cells transfected with (A) Ctrl mimics and miR-486-5p mimics and (B) Ctrl inhibitor and miR-486-5p inhibitors; representative images of apoptosis and quantitative results of apoptosis. (C) Western blotting was performed to detect cleaved-caspase3 expression in AGS and HGC27 cells. Cell cycle was detected by PI staining in AGS and HGC27 cells transfected with (D) Ctrl mimics and miR-486-5p mimics and (E) Ctrl inhibitor and miR-486-5p inhibitors. ${ }^{*} \mathrm{P}<0.05,{ }^{* *} \mathrm{P}<0.01,{ }^{* * * *} \mathrm{P}<0.001$. miR, microRNA; GC, gastric cancer; Ctrl, control; PI, propidium iodide; Ctrl, control; Cle, cleaved. 
A

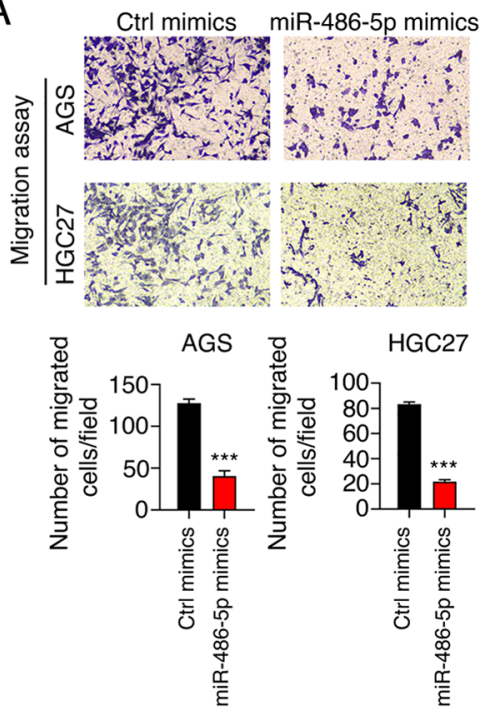

D Ctrinhibitor miR-486-5pinitho

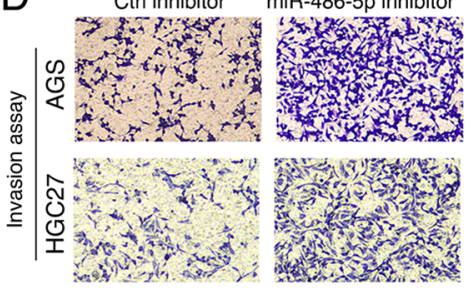

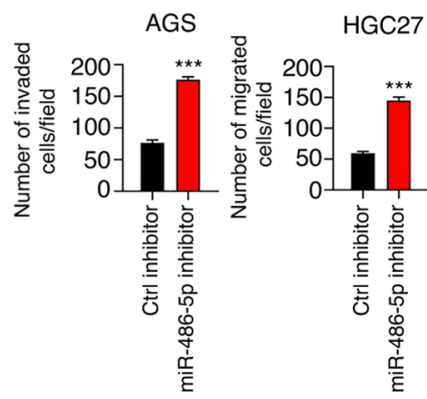

B Ctrl inhibitor miR-486-5p inhibitor
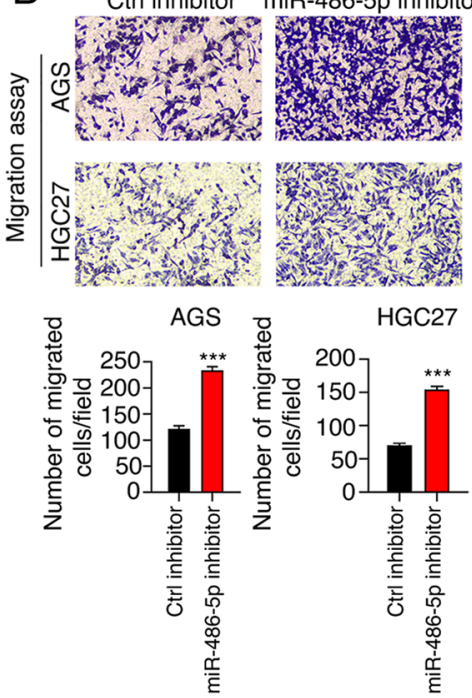

$E$

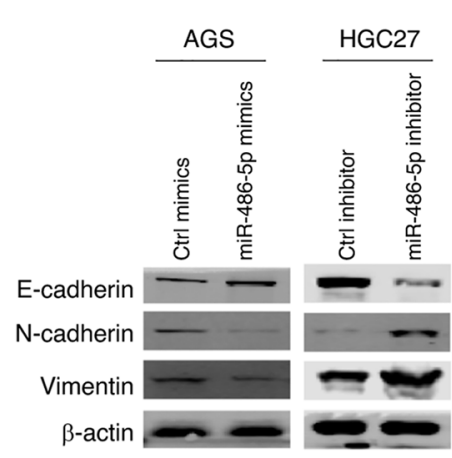

C
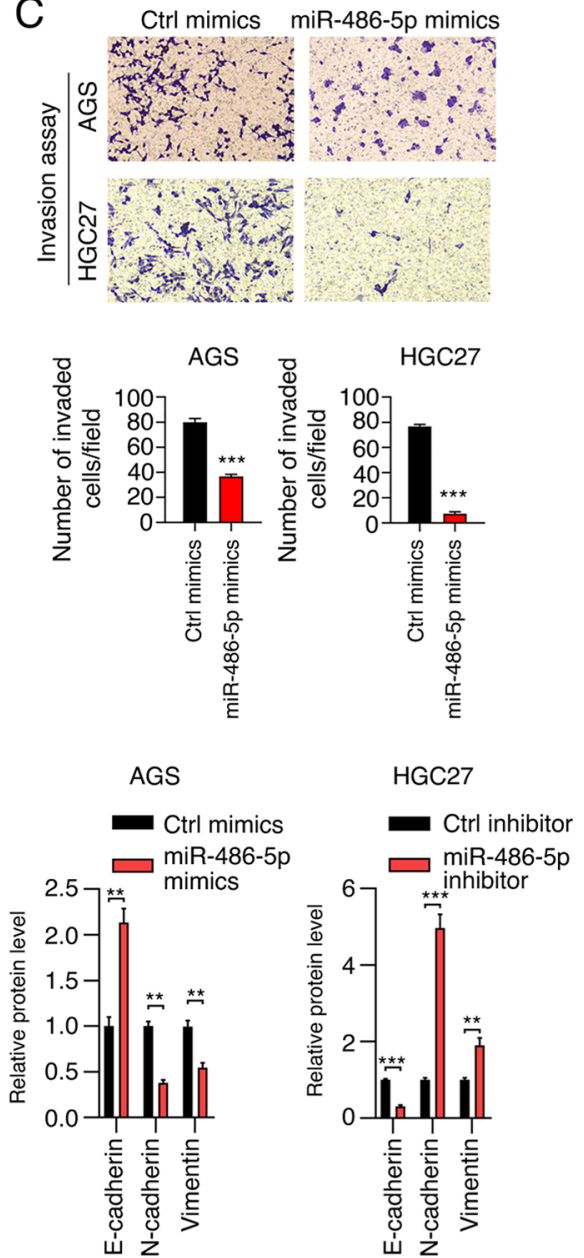

Figure 4. miR-486-5p suppresses the migration and invasion of GC cells. Cell migration was detected by Transwell assay in AGS and HGC27 cells transfected with (A) Ctrl-mimics and miR-486-5p mimics and (B) Ctrl-inhibitors and miR-486-5p inhibitors; representative images of cell migration and quantitative results of cell migration. Cell invasion was detected by Transwell assay in AGS and HGC27 cells transfected with (C) Ctrl-mimics and miR-486-5p mimics and (D) Ctrl-inhibitors and miR-486-5p inhibitors; representative images of cell invasion and quantitative results of cell invasion. (E) Western blot analysis of E-cadherin, N-cadherin and Vimentin in AGS cells transfected with Ctrl-mimics and miR-486-5p mimics and in HGC27 cells transfected with Ctrl-inhibitors and miR-486-5p inhibitors. ${ }^{* *} \mathrm{P}<0.01,{ }^{* * *} \mathrm{P}<0.001$. Magnification, $\mathrm{x} 40$. miR, microRNA; GC, gastric cancer; Ctrl, control.

of miR-486-5p contributes to lung cancer progression through targeting the proto-oncogene ARHGAP5 (33). miR-486-5p could cause deficient metastasis and EMT in prostate cancer by targeting Snail (34). Downregulation of miR-486-5p is also observed in colorectal cancer patients (35). DNA-methylation causes downregulation of miR-486-5p-stimulated colorectal cancer cell growth and migration, depending on the activation of the PLAGL2/IGF2/ $\beta$-catenin signaling pathway (36). However, there is evidence that demonstrates miR-486-5p is an onco-miRNA. miR-486-5p was highly expressed in and promoted myeloid leukemias of Down syndrome by cooperating with GATA1s (37). Although the expression of miR-486-5p is reduced in gastric adenocarcinoma patients (38), the function and molecular mechanism of miR-486-5p in GC are poorly investigated. Consistent with this finding, the present study found that miR-486-5p was downregulated in GC tissues and cells. miR-486-5p mimics and inhibitors transfection revealed that miR-486-5p suppressed cell growth, proliferation and migration in AGS and HGC27 cells. Cell cycle was arrested at $\mathrm{G}_{0} / \mathrm{G}_{1}$ phase and apoptosis was promoted by miR-486-5p. miR-486-5p also suppressed EMT in GC. These results indicated that $\mathrm{miR}-486-5 \mathrm{p}$ functioned as a tumor suppressive miRNA in GC, which was similar with the function of miR-486-5p observed in other malignancies, such as lung cancer, colorectal cancer and prostate cancer $(39,40)$.

The FGF family comprises 24 different polypeptides. FGF9 gene is mapped on chromosome 13q11-q12 and the protein is abundant in multiple mammal organs (41). Overexpression of FGF9 contributes to the development of a wide variety of types of cancer. The first evidence revealing the oncogenic function of FGF9 was reported in 1999 by Giri et al (42). Subsequently, a number of studies on cancer research provided evidence that supported the oncogenic function of FGF9. For example, FGF9 is regulated 


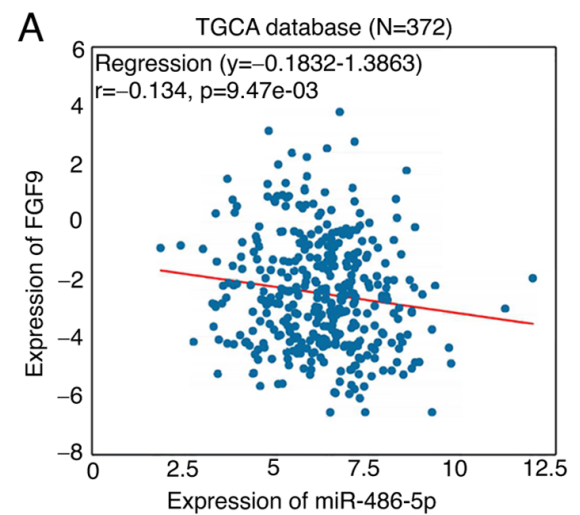

C
B

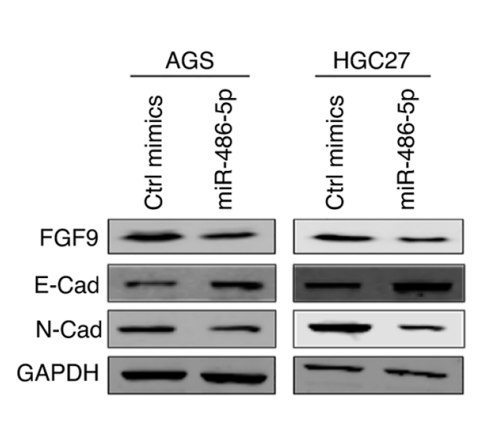

Ctrl mimics
miR-486-5p mimics

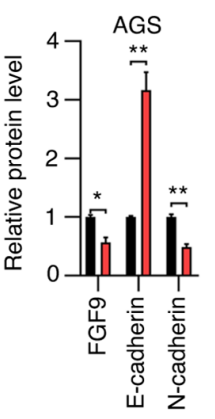

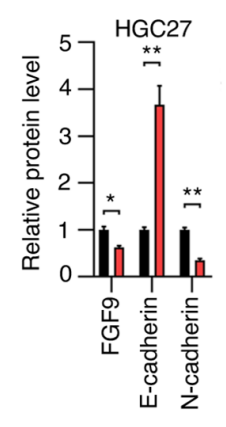

FGF9 Wlid type 3'UTR: 5'UAAUGAAACAUGUACAGGC...
hsa-miR-486-5p: 3' GAGCCCCGUCGAGUCAUGUCCU
* $* * * * * *$
FGF9 Mutant 3' UTR: 5'.. UAAUGAAACAUCAAUGCCC...

D

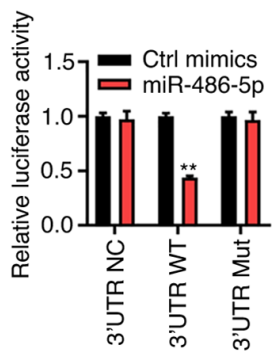

Figure 5. miR-486-5p downregulates FGF9 in GC tissues and cells. (A) Correlation between miR-486-5p expression and FGF9 expression in stomach adenocarcinoma tissues, which was analyzed from TCGA database. (B) Western blot analysis of FGF9 expression in Ctrl-mimics and miR-486-5p overexpressed AGS and HGC27 cells. (C) A putative binding site of miR-486-5p in the 3'UTR sequence of FGF9, as predicted by TargetScan. (D) Luciferase activity was detected in cells which were co-transfected with psiCHECK vectors, which were inserted with WT or MU 3'UTR sequence of FGF9 and miR-486-5p mimics. ${ }^{*} \mathrm{P}<0.05,{ }^{* *} \mathrm{P}<0.01 \mathrm{Ctrl}$ mimics vs. miR-486-50p cotransfected with WT 3'UTR sequence of FGF9. miR, microRNA; GC, gastric cancer; Ctrl, control; TCGA, The Cancer Genome Atlas database; UTR, untranslated region; WT, wild-type; MU, mutant; FGF9, fibroblast growth factor 9.

A

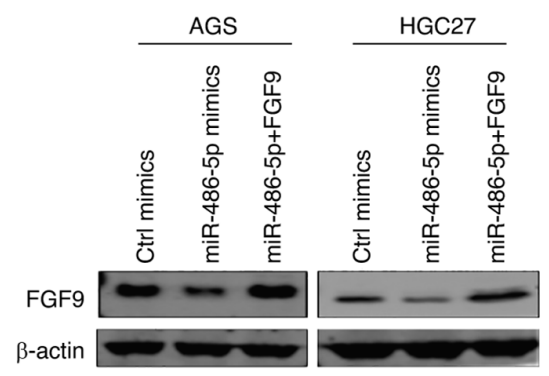

C

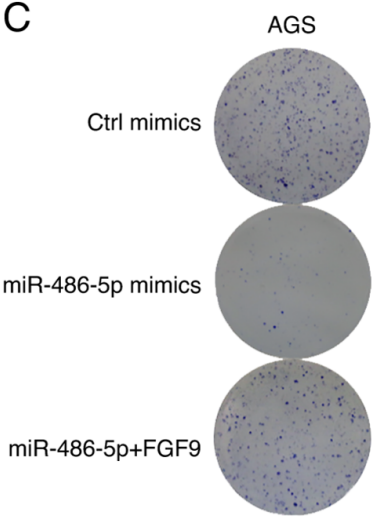

B
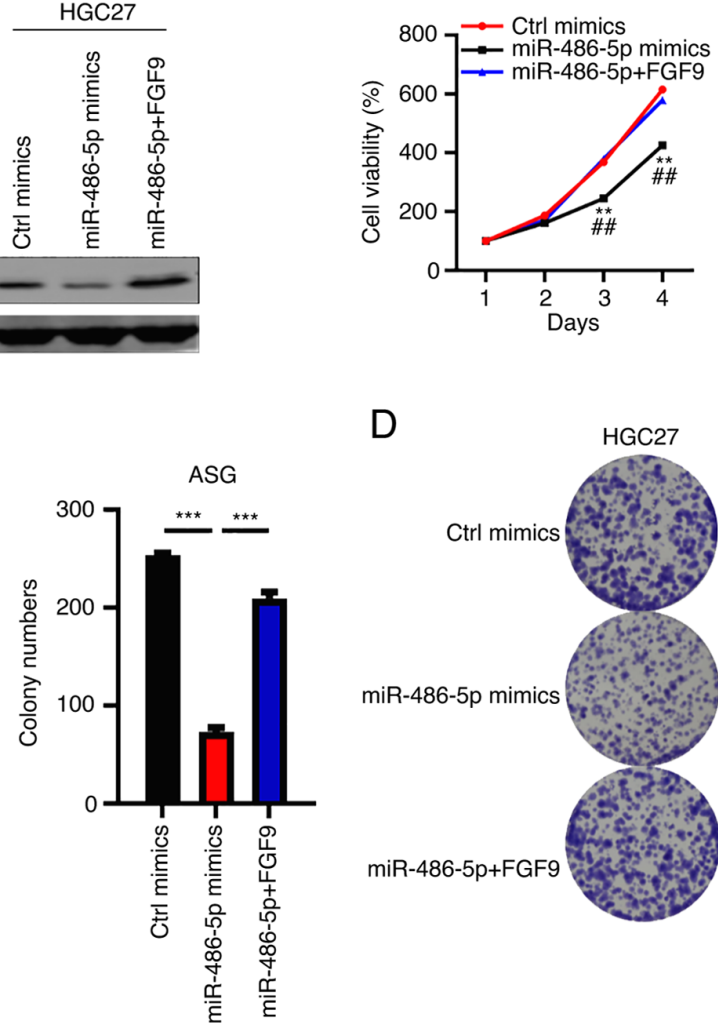

D

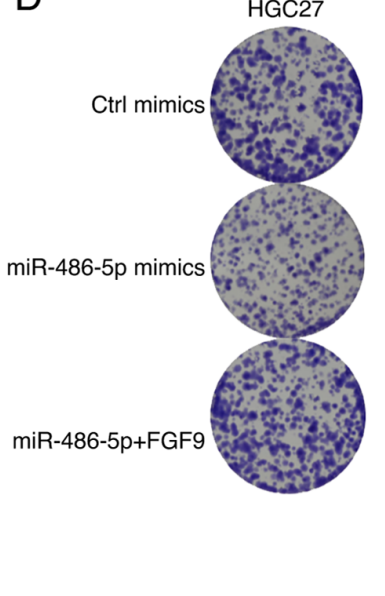

HGC27
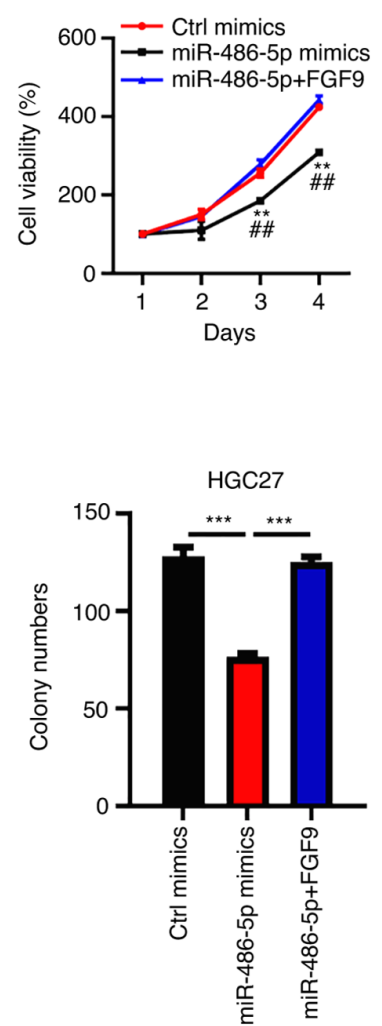

Figure 6. FGF9 reverses the function of miR-486-5p on GC cell growth. (A) Western blot analysis of FGF9 in AGS and HGC27 cells transfected with Ctrl-mimics, miR-486-5p mimics and miR-486-5p mimics + FGF9. (B) CCK8 assay was performed to detect cell growth in AGS and HGC27 cells transfected with Ctrl-mimics, miR-486-5p mimics and miR-486-5p mimics + FGF9. ${ }^{* *} \mathrm{P}<0.01$ Ctrl mimics vs. miR-485-5p mimics and ${ }^{\# \#} \mathrm{P}<0.01 \mathrm{miR}-485-5 \mathrm{p}$ mimics vs. FGF9. (C) Colony formation was assessed in AGS cells transfected with Ctrl-mimics, miR-486-5p mimics and miR-486-5p mimics + FGF9. Colony numbers were presented. ${ }^{* * * *} \mathrm{P}<0.001$. (D) Colony formation was assessed in HGC27 cells transfected with Ctrl-mimics, miR-486-5p mimics and miR-486-5p mimics + FGF9. Colony numbers were presented. ${ }^{* * *} \mathrm{P}<0.001$. FGF9, fibroblast growth factor 9; miR, microRNA; GC, gastric cancer; Ctrl, control. 
by Wnt signaling and exhibits oncogenic function in ovarian endometrioid adenocarcinomas (43). FGF9 is also induced by hypoxia and contributes to colon cancer development (44). In GC, FGF9 can be targeted by miR-26a, which is identified as a tumor suppressive miRNA (45). Nevertheless, the correlation between miR-486-5p and FGF9 remained to be elucidated. To explore the downstream targets of miR-486-5p in GC, immunoblotting and luciferase activity assays were performed and it was found that miR-486-5p could bind to the 3'UTR sequence of fibroblast growth factor 9 (FGF9). miR-486-5p downregulated FGF9 in GC cells. There was a negative correlation between miR-486-5p expression and FGF9 expression in STAD samples based on TCGA database. Notably, FGF9 ectopic expression significantly restored the proliferation and colony formation ability of AGS and HGC27 cells which had been transfected with miR-486-5p mimics. These results indicated that miR-486-5p suppresses GC growth through downregulating of FGF9.

In summary, the present study provided evidence that the miR-486-5p/FGF9 axis promoted GC cell growth and proliferation. As miR-486-5p was downregulated in GC patients, the results suggested that miR-486-5p had tumor suppressive function in GC. Targeting FGF9 might be an option for the patients with dysregulated miR-486-5p.

\section{Acknowledgements}

Not applicable.

\section{Funding}

The research was financially supported by the Heilongjiang Health and Family Planning Commission Scientific Research Project (grant no. 2018-351) and Heilongjiang Provincial Health Commission scientific research project (grant no. 2019-314).

\section{Availability of data and materials}

The datasets used and/or analyzed during the current study are available from the corresponding author upon reasonable request.

\section{Authors' contributions}

HT and QW designed the study. WW and CL performed most of the experiments and wrote the manuscript. RY and QT performed the western blot assay. HT and QW confirm the authenticity of all the raw data. All authors reviewed and approved the final manuscript.

\section{Ethics approval and consent to participate}

The experiments were carried out according to World Medical Association Declaration of Helsinki and were supported by the Ethics Committee of First Affiliated Hospital of Jiamusi University (Ethic approval number: 202067).

\section{Patient consent for publication}

Not applicable.

\section{Competing interests}

The authors declare that they have no competing interests.

\section{References}

1. Smyth E, Nilsson M, Grabsch H, van Grieken N and Lordick F: Gastric cancer. Lancet 396: 635-648, 2020.

2. Nagtegaal ID, Odze RD, Klimstra D, Paradis V, Rugge M, Schirmacher P, Washington KM, Carneiro F and Cree IA; WHO Classification of Tumours Editorial Board: The 2019 WHO classification of tumours of the digestive system. Histopathology 76: 182-188, 2020.

3. O'Connor A, O'Morain C and Ford A: Population screening and treatment of Helicobacter pylori infection. Nat Rev Gastroenterol Hepatol 14: 230-240, 2017.

4. Schulz C, Schutte K, Mayerle J and Malfertheiner P: The role of the gastric bacterial microbiome in gastric cancer: Helicobacter pylori and beyond. Therap Adv Gastroenterol 12: 1756284819894062,2019

5. Zang Z, Cutcutache I, Poon SL, Zhang SL, McPherson JR, Tao J, Rajasegaran V, Heng HL, Deng N, Gan A, et al: Exome sequencing of gastric adenocarcinoma identifies recurrent somatic mutations in cell adhesion and chromatin remodeling genes. Nat Genet 44: 570-574, 2012.

6. Sethi NS, Kikuchi O, Duronio GN, Stachler MD, McFarland JM, Ferrer-Luna R, Zhang Y, Bao C, Bronson R, Patil D, et al: Early TP53 alterations engage environmental exposures to promote gastric premalignancy in an integrative mouse model. Nat Genet 52: 219-230, 2020

7. Ebert M, Yu J, Hoffmann J, Rocco A, Röcken C, Kahmann S, Müller O, Korc M, Sung JJ and Malfertheiner P: Loss of beta-catenin expression in metastatic gastric cancer. J Clin Oncol 21: 1708-1714, 2003.

8. Zarkavelis G, Boussios S, Papadaki A, Katsanos KH, Christodoulou DK and Pentheroudakis G: Current and future biomarkers in colorectal cancer. Ann Gastroenterol 30: 613-621, 2017.

9. Esteller M: Non-coding RNAs in human disease. Nat Rev Genet 12: 861-874, 2011.

10. Rupaimoole R, Calin G, Lopez-Berestein G and Sood A: miRNA deregulation in cancer cells and the tumor microenvironment. Cancer Discov 6: 235-246, 2016.

11. Zhang Y, Wang S, Lai Q, Fang Y, Wu C, Liu Y, Li Q, Wang X, $\mathrm{Gu} \mathrm{C}$, Chen J, et al: Cancer-associated fibroblasts-derived exosomal miR-17-5p promotes colorectal cancer aggressive phenotype by initiating a RUNX3/MYC/TGF- $\beta 1$ positive feedback loop. Cancer Lett 491: 22-35, 2020.

12. Wu Q, Luo G, Yang Z, Zhu F, An Y, Shi Y and Fan D: miR-17-5p promotes proliferation by targeting SOCS6 in gastric cancer cells. FEBS Lett 588: 2055-2062, 2014.

13. Song J, Liu Y, Wang T, Li B and Zhang S: miR-17-5p promotes cellular proliferation and invasiveness by targeting RUNX3 in gastric cancer. Biomed Pharmacother 128: 110246, 2020.

14. Shi Y, Liu G, Li S and Liu X: miR-17-5p knockdown inhibits proliferation, autophagy and promotes apoptosis in thyroid cancer via targeting PTEN. Neoplasma 67: 249-258, 2020.

15. Wang J, Jia X, Liu Y, Dong JH, Ren XM, Xu O, Liu SH and Shan CG: Silencing of miR-17-5p suppresses cell proliferation and promotes cell apoptosis by directly targeting PIK3R1 in laryngeal squamous cell carcinoma. Cancer Cell Int 20: 14, 2020.

16. Wang Y, Xu W, Wang Y, Xu X, Lv S and Dong X: miR-17-5p promotes migration and invasion in breast cancer cells by repressing netrin 4. Int J Clin Exp Pathol 12: 1649-1657, 2019.

17. Wang G, Zhou Y, Chen W, Yang Y, Ye J, Ou H and Wu H: miR-21-5p promotes lung adenocarcinoma cell proliferation, migration and invasion via targeting WWC2. Cancer Biomark 28: $549-559,2020$.

18. Xiao T and Jie Z: MiR-21 promotes the invasion and metastasis of gastric cancer cells by activating epithelial-mesenchymal transition. Eur Surg Res 60: 208-218, 2019.

19. Sun L, Tian D, Yang Z and Li J: Exosomal miR-21 promotes proliferation, invasion and therapy resistance of colon adenocarcinoma cells through its target PDCD4. Sci Rep 10: 8271, 2020.

20. Kim K, Kim HH, Lee CH, Kim S, Cheon GJ, Kang KW, Chung JK and Youn H: Therapeutic efficacy of modified anti-miR21 in metastatic prostate cancer. Biochem Biophys Res Commun 529: 707-713, 2020. 
21. Boussios S, Ozturk MA, Moschetta M, Karathanasi A Zakynthinakis-Kyriakou N, Katsanos KH, Christodoulou DK and Pavlidis N: The developing story of predictive biomarkers in colorectal cancer. J Pers Med 9: 12, 2019.

22. Valenti MT, Deiana M, Cheri S, Dotta M,Zamboni F, Gabbiani D, Schena F, Dalle Carbonare L and Mottes M: Physical exercise modulates miR-21-5p, miR-129-5p, miR-378-5p, and miR-188-5p expression in progenitor cells promoting osteogenesis. Cells 8 : $742,2019$.

23. Boussios S, Abson C, Moschetta M, Rassy E, Karathanasi A, Bhat T, Ghumman F, Sheriff M and Pavlidis N: Poly (ADP-Ribose) polymerase inhibitors: Talazoparib in ovarian cancer and beyond. Drugs R D 20: 55-73, 2020.

24. Pang W, Tian X, Bai F, Han R, Wang J, Shen H, Zhang X, Liu Y, Yan X, Jiang F and Xing L: Pim-1 kinase is a target of miR-486-5p and eukaryotic translation initiation factor 4E, and plays a critical role in lung cancer. Mol Cancer 13: 240, 2014.

25. Rask L, Balslev E, Søkilde R, Høgdall E, Flyger H, Eriksen J and Litman T: Differential expression of miR-139, miR-486 and miR-21 in breast cancer patients sub-classified according to lymph node status. Cell Oncol (Dordr) 37: 215-227, 2014.

26. Ma X, Wei J, Zhang L, Deng D, Liu L, Mei X, He X and Tian J: miR-486-5p inhibits cell growth of papillary thyroid carcinoma by targeting fibrillin-1. Biomed Pharmacother 80: 220-226, 2016

27. Livak KJ and Schmittgen TD: Analysis of relative gene expression data using real-time quantitative PCR and the 2(-Delta Delta C(T)) method. Methods 25: 402-408, 2001.

28. Wang RA, Li ZS, Yan QG, Bian XW, Ding YQ, Du X, Sun BC, Sun YT and Zhang XH: Resistance to apoptosis should not be taken as a hallmark of cancer. Chin J Cancer 33: 47-50, 2014

29. Hu H, Xu H, Lu F, Zhang J, Xu L, Xu S, Jiang H, Zeng Q Chen $\mathrm{E}$ and $\mathrm{He} \mathrm{Z}$ : Exosome-derived miR-486-5p regulates cell cycle, proliferation and metastasis in lung adenocarcinoma via targeting NEK2. Front Bioeng Biotechnol 8: 259, 2020.

30. Saragoni L: Upgrading the definition of early gastric cancer: Better staging means more appropriate treatment. Cancer Biol Med 12: 355-361, 2015.

31. Yeung KT and Yang J: Epithelial-mesenchymal transition in tumor metastasis. Mol Oncol 11: 28-39, 2017.

32. Chang MM, Lai MS, Hong SY, Pan BS, Huang H, Yang SH, Wu CC, Sun HS, Chuang JI, Wang CY and Huang BM: FGF9/FGFR2 increase cell proliferation by activating ERK1/2, $\mathrm{Rb} / \mathrm{E} 2 \mathrm{~F} 1$, and cell cycle pathways in mouse Leydig tumor cells. Cancer Sci 109: 3503-3518, 2018.

33. Wang J, Tian X, Han R, Zhang X, Wang X, Shen H, Xue L, Liu Y, Yan X, Shen J, et al: Downregulation of miR-486-5p contributes to tumor progression and metastasis by targeting protumorigenic ARHGAP5 in lung cancer. Oncogene 33: 1181-1189, 2014.

34. Zhang X, Zhang T, Yang K, Zhang M and Wang K: miR-486-5p suppresses prostate cancer metastasis by targeting Snail and regulating epithelial-mesenchymal transition. Onco Targets Ther 9: 6909-6914, 2016
35. Shindo Y, Hazama S, Nakamura Y, Inoue Y, Kanekiyo S, Suzuki N, Takenouchi H, Tsunedomi R, Nakajima M, Ueno T, et al: miR-196b, miR-378a and miR-486 are predictive biomarkers for the efficacy of vaccine treatment in colorectal cancer. Oncol Lett 14: 1355-1362, 2017.

36. Liu X, Chen X, Zeng K, Xu M, He B, Pan Y, Sun H, Pan B, Xu X, $\mathrm{Xu}$, et al: DNA-methylation-mediated silencing of miR-486-5p promotes colorectal cancer proliferation and migration through activation of PLAGL2/IGF2/ $\beta$-catenin signal pathways. Cell Death Dis 9: 1037, 2018

37. Shaham L, Vendramini E, Ge Y, Goren Y, Birger Y, Tijssen MR, McNulty M, Geron I, Schwartzman O, Goldberg L, et al: MicroRNA-486-5p is an erythroid oncomiR of the myeloid leukemias of Down syndrome. Blood 125: 1292-1301, 2015.

38. Chen H, Ren C, Han C, Wang D, Chen Y and Fu D: Expression and prognostic value of miR-486-5p in patients with gastric adenocarcinoma. PLoS One 10: e0119384, 2015.

39. Shao Y, Shen YQ, Li YL, Liang C, Zhang BJ, Lu SD, He YY, Wang P, Sun QL, Jin YX and Ma ZL: Direct repression of the oncogene CDK4 by the tumor suppressor miR-486-5p in non-small cell lung cancer. Oncotarget 7: 34011-34021, 2016.

40. Pisano A, Grinan-Lison C,Farace C, Fiorito G,Fenu G, Jiménez G, Scognamillo F, Peña-Martin J, Naccarati A, Pröll J, et al: The inhibitory role of miR-486-5p on CSC phenotype has diagnostic and prognostic potential in colorectal cancer. Cancers (Basel) 12: 3432,2020

41. Mattei MG, Penault-Llorca F, Coulier F and Birnbaum D: The HumanFGF9Gene Maps to chromosomal region 13q11-q12. Genomics 29: 811-812, 1995.

42. Giri D, Ropiquet F and Ittmann M: FGF9 is an autocrine and paracrine prostatic growth factor expressed by prostatic stromal cells. J Cell Physiol 180: 53-60, 1999.

43. Hendrix N, Wu R, Kuick R, Schwartz D, Fearon E and Cho K: Fibroblast growth factor 9 has oncogenic activity and is a downstream target of Wnt signaling in ovarian endometrioid adenocarcinomas. Cancer Res 66: 1354-1362, 2006.

44. Chen TM, Shih YH, Tseng JT, Lai MC, Wu CH, Li YH, Tsai SJ and Sun HS: Overexpression of FGF9 in colon cancer cells is mediated by hypoxia-induced translational activation. Nucleic Acids Res 42: 2932-2944, 2014

45. Deng M, Tang HL, Lu XH, Liu MY, Lu XM, Gu YX, Liu JF and He ZM: miR-26a suppresses tumor growth and metastasis by targeting FGF9 in gastric cancer. PLoS One 8: e72662, 2013.

This work is licensed under a Creative Commons Attribution-NonCommercial-NoDerivatives 4.0 International (CC BY-NC-ND 4.0) License. 